\title{
AMCP's Annual Meeting: Controversies Surrounding Helicobacter pylori
}

Numerous sessions at the AMCP convention in San Francisco focused on practical information about medications. The diagnosis and treatment of Helicobacter pyloriinfection are explored in this feature article.
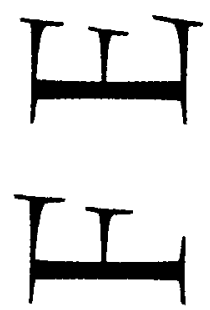

$\pi$ ttendees at AMCP's Eighth Annual Meeting left San Francisco with no shortage of information and ideas on medications and ways to manage them. The recognition of the role played by Helicobacter pylori in gastric and duodenal ulcers and oncolytic conditions were explored in a Saturday morning session. The program was supported by Abbott Laboratories.

\section{BACTERIOLOGY} REVOLUTIONIZES ACID PEPTIC DISEASE

"No acid, no ulcer." That dictum, L. MICHAEL POSEY

L. MICHAEL POSEY is Editor, JMCP.

Copyright @ 1996 Academy of Managed Care Pharmacy, Inc. All rights reserved. once the basis of thought on treatment of peptic ulcer disease and other acid peptic disorders, has been discarded as the role of $\mathrm{H}$. pylori in the disease has become clear over the past 10 years. 
Table 1. Patient Selection for H. pylori Eradication Therapy

\section{$\Delta$ Who to treat:}

Patients with documented $H$. pylori infections and these clinical conditions:

Active duodenal ulcer

Active gastric ulcer

Past history of gastric or duodenal ulcer

MALT lymphoma

\section{Who not to treat:}

Patients with undocumented H. pylori

Asymptomatic individuals

General population for prevention of gastric cancer

That message, by Larry Danziger, Pharm.D., and Jay Goldstein, M.D., of the University of Illinois at Chicago, was brought home for listeners by the realization that $H$. pylori infection is now "the most common infectious disease throughout the world today."

\section{THE ORGANISM AND ITS EFFECTS}

The H. pylori organism is very well adapted to life in the acidic environment of the upper gastrointestinal tract, Danziger explained. Spiral shaped, the organism has two to six flagella providing motility, is microaerophilic, and commits $10 \%$ of its protein and energy resources to urease, which splits urea into the basic ammonia and bicarbonate ions. H. pylori colonizes the mucous layer over the gastric epithelium only, more commonly in the antrum rather than the body of the stomach. The infection leads to an acute and chronic inflammatory response.

The prevalence of $H$. pylori infection increases with age; some $75 \%$ of patients over age 65 years are infected. Spread of the organism is thought to be through the fecal-oral route between family members and those sleeping in the same bed. The prevalence of H. pylori infection in patients with specific disorders reads this way:

A $100 \%$ of those with chronic active gastritis

A About $95 \%$ of patients with duode- nal ulcers

A About $70 \%$ of those with gastric ulcers Additionally, acid secretion from chronic H. pylori infection has been linked to development of gastric metaplasia in the duodenum.

\section{DIAGNOSIS: BALANCING COSTS AND RESULTS}

Three choices are available for diagnosis, according to Goldstein. Culture of $H$. pylori is not cost effective, being difficult and expensive to perform and taking seven days for results.

Histology has been the mainstay of diagnosis. The gold standard continues to be staining endoscopic samples using hematoxylin and eosin. Even though the test costs $\$ 250$, Goldstein advocates its use, since it is being added to a $\$ 1,000$ endoscopic procedure and produces a definitive diagnosis.

Serology is an option for initial diagnosis, but it fails to differentiate between active and treated or prior infection. It is inexpensive and highly sensitive and specific, Goldstein explained. ELISA tests are quantitative, while office-based procedures are qualitative and provide results in four minutes.

More recently available are urea breath tests, which take advantage of the organisms high level of urease production. Just approved by FDA this spring, the tests use urea labeled with radioactive carbon $\left({ }^{13} \mathrm{C}\right.$ or $\left.{ }^{14} \mathrm{C}\right)$. The patient takes the radiolabeled urea orally, and 20 minutes later the presence of radiolabeled carbon dioxide in the breath is measured. The test, highly sensitive and specific, confirms active infection anywhere in the stomach, and costs about $\$ 200-250$.

\section{CONTROVERSIES ON WHOM TO TREAT}

Goldstein then jumped into the sticky issue of treatment of documented H. pylori infections. "We are not going to eradicate the organism," he began, giving the lists of definitive candidates and noncandidates shown in Table 1. Four areas of controversy create most current discussions about when and when not to treat $H$. pylori:

A Helicobacter-positive dyspepsia

$\Delta$ Helicobacter-positive gastritis

$\Delta$ Individuals with a first-degree relative with gastric cancer

$\Delta$ Individuals with a spouse/significant other with an ulcer

The controversy surrounding dyspepsia was explored in detail in the session.

Endoscopy can differentiate among the five types of patients with dyspepsia:

A Duodenal ulcer (20\%)

A Gastric ulcer (5\%)

$\Delta$ Gastritis (40\%)

$\Delta$ Other (including reflux disease, 20\%)

$\Delta \operatorname{Normal}(15 \%)$

Therapy for H. pylori will not aid patients in the last two categories, while those in the first two groups definitely should receive treatment. For patients under age 45 , a noninvasive test can be used to aid differentiation. Serology or urea breath tests, if positive, lead to a need for endoscopy and/or anti-H. pylori treatment. Patients with negative results can be treated empirically with acid-inhibition therapy.

The main point to remember, Goldstein said, is this: "If you're going to test, you have to treat." Here are his 
Table 2. Common H. pylori Eradication Regimens

\begin{tabular}{|c|c|}
\hline \multicolumn{2}{|c|}{ Bismuth triple therapy (14 days; $90 \%$ eradication rate) } \\
\hline Bismuth (PeptoBismol) & 2 tabs p.o. q.i.d. \\
\hline Metronidazole & 250 mg p.o. q.i.d. \\
\hline Tetracycline & 500 mg p.o. q.i.d. \\
\hline \multicolumn{2}{|c|}{ PPI dual therapy (14 days; $80 \%$ eradication rate) } \\
\hline Omeprazole & $40 \mathrm{mg}$ p.o. q.d. \\
\hline Clarithromycin & $500 \mathrm{mg}$ p.o. t.i.d. \\
\hline \multicolumn{2}{|c|}{ PPI triple therapy ( 14 or fewer days; $90 \%$ eradication rate) } \\
\hline Omeprazole & 40 mg p.o. q.d. \\
\hline Clarithromycin & 500 mg p.o. t.i.d. \\
\hline PLUS & \\
\hline Metronidazole & $500 \mathrm{mg}$ p.o. b.i.d. \\
\hline OR & \\
\hline Amoxicillin & 1,000 mg p.o. b.i.d. \\
\hline \multicolumn{2}{|c|}{ Combination therapy with $\mathrm{RBC}(80 \%$ eradication rate) } \\
\hline Ranitidine/bismuth citrate & $400 \mathrm{mg}$ p.o. b.i.d. $\times 28$ days \\
\hline Clarithromycin & $500 \mathrm{mg}$ p.o. t.i.d. $\times 14$ days \\
\hline
\end{tabular}

thoughts on the best regimens to use for that.

\section{ANTI-H. PYLORI PROTOCOLS}

Table 2 lists the four most common H. pylori protocols. Goldstein emphasized two general treatment guidelines:

A Monotherapy is unacceptable: it's just going to produce resistance and treatment failures

$\Delta$ Do not substitute one agent for another (e.g., ampicillin for amoxicillin; azithromycin or erythromycin for clarithromycin)

In addition to bacterial resistance, three other factors influence patient outcomes: innate efficacy, compliance, and side effects. Compliance is a particular problem with $H$. pylori therapy, partially because of the complexity and length of the regimens and also because of the agents' side effects. When the compliance rate with bismuth triple therapy exceeds $60 \%$, the eradication rate if $95 \%$. However, Goldstein noted, it falls to $69 \%$ with lower compliance.

Additionally, metronidazole resistance decreases the efficacy of bismuth triple therapy by 35-65\%.

Regimens such as omeprazole plus clarithromycin are much simpler, requiring four tablets/day versus 16 with bismuth triple therapy. The combination of clarithromycin and omeprazole is based on the effects on stomach $\mathrm{pH}$ of the antisecretory agent. Proton-pump inhibitors increase the stomach $\mathrm{pH}$, allowing clarithromycin to become more concentrated in the mucosal lining where $H$. pylori colonizes.

After eradication, reinfection rates are quite low, explained Goldstein. Rates per patient-year have ranged from 0.6 to $2.3 \%$. "For each month the patient remains symptom-free," Goldstein summarized, "is one less month of $\mathrm{H}_{2}$ antagonist therapy under the old schools of thought. Even if the patient
Table 3. Two-Year Direct Cost Model for Duodenal Ulcers

\begin{tabular}{lr}
\hline \multicolumn{2}{c}{ Cost (\$) } \\
\hline Therapy & 720 \\
\hline Bismuth triple therapy & 769 \\
Omeprazole/clarithromycin & 1028 \\
Omeprazole/amoxicillin $^{a}$ & 1701 \\
$\mathrm{H}_{2}$ antagonist maintenance & \\
therapy & \\
\hline a Therapy no longer considered effective.
\end{tabular}

has other risk factors - such as concomitant therapy with nonsteroidal anti-inflammatory agents, the H. pylori status needs to be determined and the patient treated if positive. The avoidance of years of symptomatic therapy will be cost-effective despite the high one-time costs." He provided the cost data in Table 3 in support of his position; the data are based on a two-year period, which Goldstein said was the average length of coverage in a managed care plan.

\section{MICROBE CHANGES THE WORLD OF ULCER THERAPY}

With $H$. pylori now firmly established as an etiologic agent for peptic ulcer disease, and the recent recognition of a role for Chlamydia pneumoniae in pathogenesis of atherosclerosis (see June Journal of the American College of Cardiology), people are wondering what else will turn out to be caused by invisible but ubiquitous bacteria. Certainly, the recognition of the key role played by $H$. pylori has altered completely basic approaches to diagnosis and management of dyspepsia, ulcer, and oncolytic conditions of the upper gastrointestinal tract. 